\title{
(Dis-)simulation et fiction dans le roman «Ich» de Wolfgang Hilbig (1993)
}

(Dis-)simulation und Fiktion: Der Roman „Ich“von Wolfgang Hilbig (1993)

(Dis-)simulation and fiction in the novel "Ich" (1993) by Wolfgang Hilbig

\section{Christian Klein}

\section{(2) OpenEdition}

Journals

Édition électronique

URL : http://journals.openedition.org/ceg/1374

DOI : $10.4000 /$ ceg. 1374

ISSN : 2605-8359

Éditeur

Presses Universitaires de Provence

Édition imprimée

Date de publication : 15 juin 2015

Pagination : 21-30

ISBN : 978-2-85399-993-9

ISSN : 0751-4239

\section{Référence électronique}

Christian Klein, « (Dis-)simulation et fiction dans le roman «Ich» de Wolfgang Hilbig (1993) », Cahiers d'Études Germaniques [En ligne], 68 | 2015, mis en ligne le 17 décembre 2017, consulté le 05 décembre 2020. URL : http://journals.openedition.org/ceg/1374; DOI : https://doi.org/10.4000/ceg.1374 


\section{(Dis-)simulation et fiction dans le roman " Ich " de Wolfgang Hilbig (1993)}

Christian KLEIN

Université Paris Ouest Nanterre La Défense

En 1993 paraît un roman de Wolfgang Hilbig au titre à double entrée, "Moi » ( Ich»), sur les mésaventures identitaires d'un écrivain-ouvrier de RDA qui a été recruté par la Sécurité (Stasi) pour espionner le milieu des écrivains de la scène alternative du Prenzlauer Berg à Berlin dans les années qui ont précédé l'effondrement du socialisme d'État en 1989. Hilbig s'inspire de faits réels. Depuis que Wolf Biermann accusa publiquement, en 1991, l'écrivain dissident Sascha Anderson, chef de file du Prenzlauer Berg, d'avoir travaillé pour la Stasi (IM-David Menzer), une polémique nationale s'est emparée des médias. L'affaire donne lieu à des révélations en cascade dans la presse sur d'autres représentants de la culture parallèle comme Rainer Schedlinski (IM-Gerhard), Ibrahim Böhme (IM-Maximilian), etc. Chaque semaine, jusqu'en 1993 et au-delà, les journaux publient des interviews, des plaidoyers, des prises de position morales ou politiques ${ }^{1}$.

L'auteur, Wolfgang Hilbig, ouvrier autodidacte né en 1941 dans une région minière près de Leipzig, a travaillé comme outilleur, comme chauffeur dans une fonderie, puis dans une blanchisserie industrielle. Très tôt il écrit des nouvelles, puis des poèmes. Il écrit la nuit sur son lieu de travail, sur la table de cuisine chez sa mère et mène une double existence difficile. Repéré par son entreprise, il est envoyé en 1964 dans un " cercle d'ouvriers-écrivains » dont il est rapidement exclu. Ses textes, qui sont nourris entre autres par la lecture des romantiques allemands, d'Edgar Poe, de Rimbaud, ne correspondent pas aux canons du réalisme socialiste et sont refusés par les revues et les éditeurs officiels. Il fréquente les scènes alternatives de Leipzig, plus tard de Berlin. En 1976, paraissent sept de ses poèmes dans la revue ouest-allemande $L$ 76. En 1977, Karl Corino lit certains de ses poèmes au Hessischer Rundfunk. Le lecteur des éditions Fischer, Thomas Beckerman, le contacte. En décembre 1977, Hilbig lui fait parvenir un premier envoi de poèmes et ils se rencontrent en mars 1978 à la foire de Leipzig ${ }^{2}$. Mais le 9 mai Hilbig est arrêté sous prétexte qu'il aurait mis le feu à un drapeau national. Il sera libéré deux mois plus tard après avoir refusé de travailler pour la Stasi. Il existe trois textes de Hilbig sur cet événement: les deux premiers sont intégrés dans un récit fictionnel, en 1989

\footnotetext{
1 Cf. bibliographie détaillée in Peter Böthig, Klaus Michael, MachtSpiele, Leipzig, Reclam Verlag, p. 392-413.

2 Cf. Thomas Beckermann, « Eigenwillige Ankunft », in Uwe Wittstock (dir.), Wolfgang Hilbig. Materialien zu Leben und Werk, Frankfurt a. M., Fischer Verlag, 1994, p. 93-113.
} 
dans le roman Eine Übertragung («Une métaphore ») ${ }^{3}$, et en 1993 dans le roman qui nous occupe aujourd'hui, «Ich $\|^{4}$. Le troisième est un témoignage personnel dans le cadre des Conférences de Francfort (Frankfurter Vorlesungen) en mai $1995^{5}$.

Dans ce dernier, Hilbig témoigne:

Am 9 Mai 1978 wurde ich [...] unter einem Vorwand verhaftet [...]. Zur Aufklärung des Verdachts, der den Grund meiner Festnahme abgegeben hatte, wurde ich im Verlauf meiner Haft so gut wie nie verhört, sehr schnell wurde mir klar, daß meine Vernehmer Angehörige der Staatssicherheit waren, denen es um die Umstände ging, unter welchen der Kontakt zu meinem Verlag in Frankfurt am Main für mich zustande gekommen war.

On lui propose un marché :

Schließlich redeten meine Vernehmer Klartext, und aus dem Ganzen wurde ein Anwerbungsversuch [...]. Man sagte mir, was ich vorhabe, unter Umgehung aller Vorschriften und gesetzlichen Genehmigungsverfahren, sei kriminell und subversiv, die Höchststrafe dafür [...] betrage fünf Jahre Gefängnis.

En échange de sa libération immédiate, il pourra publier son recueil à l'Ouest. Mais à une petite condition: il devra informer « une personne dont on lui donnera le nom » sur tout ce qui se passe ${ }^{6}$. S'il accepte, le ministère de la Sécurité d'État (MfS) lui assure le statut d'écrivain, des revenus conséquents et tous les avantages des artistes officiels : l'autorisation de circuler à l'Ouest, un logement confortable et toutes facilités pour publier en RDA.

En cas de refus, il ne serait jamais publié en RDA. Il refuse.

Derrière le prétexte de l'arrestation se dissimulait le véritable motif d'une opération de recrutement d'un nouvel agent de renseignement au service de la Sécurité d'État. Ce qui trouble l'écrivain-ouvrier ${ }^{7}$ Hilbig, c'est d'avoir été choisi pour servir d'informateur:

Als es mir passierte, fragte ich mich besorgt, was habe ich an mir, daß sie ausgerechnet mich wollen, ich selber war mir immer denkbar ungeeignet für ihre Zwecke vorgekommen. Aber sie schienen da anderer Meinung zu sein, woraus ich schloß, es müsse an meiner Situation liegen. ${ }^{8}$

Le roman "Ich» est né de cette interrogation, comme « une réflexion expérimentale » (« Gedankenexperiment $»)$.

On notera que Hilbig ne se positionnera pas d'un point de vue moral et qu'il n'a pas participé à la controverse publique sur les IM - même s'il a formulé ses reproches par ailleurs dans un échange de correspondance avec Schedlinski ${ }^{10}$.

3 Wolfgang Hilbig, Eine Übertragung [1989], Frankfurt a. M., Fischer Verlag, 2003, p. 85-125.

4 Wolfgang HiLbig, «Ich », Frankfurt a. M., Fischer Verlag, 1993. Dans la suite, les références à ce roman figureront entre parenthèses dans le corps du texte, sous la forme: «Ich » page(s) concernée(s).

5 Wolfgang Hilbig, Abriss der Kritik, Frankfurt a. M., Fischer Verlag, 1995, p. 76-81.

6 « [I]ch müsse nur eine bestimmte Person, die mir noch genannt werde, ganz genau über alle Abläufe informieren ». Ibid.

7 Nous désignons ainsi le double statut de Hilbig. Hilbig, quant à lui, récusait à son sujet la désignation officielle d' « ouvrier-écrivain » (« Arbeiterschriftsteller »).

8 Hilbig, Abriss der Kritik, p. 81.

9 Ibid.

${ }^{10}$ Cf. Stephan PABst, «"Leere Signifikanz”. Hilbigs Kritik des Poststrukturalismus », in Frédéric 


\section{L'univers diégétique du système : le mensonge d'État}

On retrouve cet événement - transformé et fictionnalisé - comme base du contrat narratif du roman "Ich». Le narrateur, W., qui vit dans une petite ville près de Leipzig, partage son temps entre les sous-sols d'une chaufferie d'usine et les bars de la ville où se retrouve un réseau d'asociaux en rupture avec les autorités. Dans ses temps libres, au travail, à la cuisine, il écrit en secret des textes littéraires - ignorés de tous: une double existence imposée par un système où toute littérature qui sort des canons du réalisme socialiste est interdite de publication.

Jusqu'au jour où il est convoqué par la mairie et où la Stasi lui fait signer au bout de convocations répétées, malgré ses dénégations, par lassitude, une reconnaissance fictive de paternité qui le lie comme informateur IM ( $I$ Ich »95-107). Tout commence donc par un mensonge d'État: alors qu'il proteste n'avoir jamais couché avec cette jeune femme, Cindy, qui passait de main en main mais jamais dans les siennes, et qu'il met les autorités au défi de prouver sa responsabilité, la Sécurité prétend fabriquer les preuves dont elle a besoin:

Glauben Sie wirklich, wir könnten Ihnen das Kind nicht beweisen? Das können wir, selbst wenn Sie in Ihrem Leben noch nie eine Frau niedergemacht haben. («Ich» 105)

Libre d'abord d'aller et venir comme avant, de continuer son travail à l'usine, et de s'adonner à ses travaux d'écriture, libre dans un premier temps de toute contrepartie concrète, le narrateur est pris progressivement dans les rets de la Firme. Il s'enfuit à Berlin, où la Sécurité le retrouve. Il y fréquente et espionne les artistes de la scène alternative du Prenzlauer Berg et continue d'écrire. Malgré des moments de confusion identitaire, la perte récurrente des repères temporels et spatiaux, il fournit des renseignements qui oscillent, mêlant les genres, entre le rapport de police et l'essai littéraire. Il publie aussi, avec le soutien plus ou moins opaque de la Stasi, des textes hybrides, médiocres, fragmentés, dans des revues alternatives.

Deux espaces diégétiques se croisent et se contaminent. Deux impostures structurent le récit sous le signe de la dis-simulation et de la simulation.

Il y a d'abord la parole officielle qui, on l'a vu, invente une représentation de la réalité. Le récit emprunte explicitement la notion de « simulation » aux écrits de Jean Baudrillard. Les textes de Baudrillard circulaient au sein de la scène est-allemande. Hilbig, qui fréquentait irrégulièrement les milieux alternatifs, connaissait aussi les principaux écrits de Baudrillard, en particulier L'Échange symbolique et la Mort ${ }^{11}$, dont un extrait du chapitre 2 est reproduit dès 1978 avec plusieurs autres articles du même auteur dans le petit volume du Merve Verlag - qui circulait en sous-main en RDA sous le titre Kool Killer oder der Aufstand der Zeichen ${ }^{12}$. Les thèses de

TeInturier, Bénédicte Terrisse, « Ich » de Wolfgang Hilbig. Littérature, identité et faux-semblants, Paris, L'Harmattan, 2013, p. 127.

11 Jean Baudrillard, L'Échange symbolique et la Mort, Paris, Gallimard, 1976 (en allemand: Der symbolische Tausch und der Tod, München, Matthes \& Seitz, 1982).

12 Jean Baudrillard, Kool Killer oder der Aufstand der Zeichen, Berlin, Merve Verlag, 1978. Le roman y emprunte explicitement, p. 34, l'expression de « signifiant vide » (BAUDRILLARD, L'Échange symbolique, p. 122 : cf. Baudrillard, Kool Killer, p. 26). 
Baudrillard sur la «simulation » fournissaient aux intellectuels de l'underground est-allemand - au prix d'une lecture singulière transposant les analyses de Baudrillard aux sociétés socialistes - un modèle interprétatif du décalage qu'ils dénoncent entre les discours officiels du SED, médias et institutions comprises, sur les acquis et les objectifs de la révolution socialiste, et la réalité quotidienne des citoyens.

Pour Baudrillard, en effet, il s'est opéré, à la suite de la dernière révolution technologique, une déconnexion entre la représentation du réel et le réel, une suspension du principe d'équivalence du signe et du réel, qui conduit à « la négation radicale du signe comme valeur ${ }^{13} »$. Nous ne sommes plus alors dans «l'ordre de l'apparence », mais dans celui de la « simulation ${ }^{14} »$, c'est-à-dire d'une « substitution au réel des signes du réel ${ }^{15} »$.

En d'autres termes, dans le domaine de la communication, tous les signes sont simulation, c'est-à-dire interchangeables entre eux, mais non échangeables contre quelque chose de réel ${ }^{16}$. Schedlinski, chef de file de la Scène berlinoise, écrit encore en 1992, à propos de ces années 80: «C'était l'ère postmoderne de l'interchangeabilité des signes ${ }^{17}$. »

Le postulat de l'érosion de la signification par son excès peut se lire comme un discrédit radical du discours politique des dirigeants de RDA en raison même de la surcharge idéologique des mots, et d'une représentation qui fonctionne comme un " simulacre ", dont la fonction est de masquer l'absence de tout référent. «Simulacre» (en allemand « Trugbild $»{ }^{18}$ ) est précisément le terme qu'utilise le narrateur pour désigner le jargon des officiers de la Sécurité en prenant pour exemple quelques lignes prélevées dans un document interne du MfS:

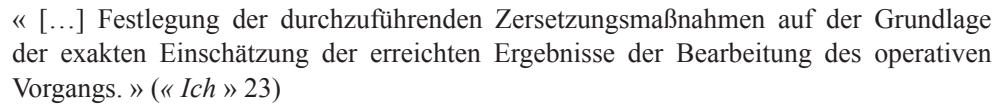

Une telle expansion potentiellement illimitée de bases nominales suspend la syntaxe prédicative dans « un enchaînement monstrueux d'abstractions » («Ich» 23). Elle dissout le sujet d'une action et, note le narrateur, « mine la réalité avec ce simulacre ${ }^{19} »$. Ainsi la parole officielle ne décrit pas la réalité politique, elle se substitue à elle, échappant alors à toute confrontation. Elle institue le mensonge comme invention du réel, comme vérité unique, dont elle prétend être dépositaire. Telle est la doxa du MfS exposée par l'officier Feuerbach, qui est chargé de contrôler les faits et gestes du narrateur: « Nous sommes en possession de l'unique vérité ${ }^{20}$. »

13 Jean BaUdrillard, Simulacres et simulation, Paris, Galilée, 1981, p. 16.

${ }_{14}$ Ibid., p. 17.

15 Ibid., p. 11.

16 Jean Baudrillard, "Politik und Simulation », in Baudrillard, Kool Killer, p. 39. Nous renvoyons au texte original publié dans Le Monde Diplomatique de mai 1978, p. 28 : « Le politique et la simulation. »

17 « « Es war das postmoderne Zeitalter der austauschbaren Zeichen » (Rainer SCHEDLINSKI, « Die Unzuständigkeit der Macht », Neue Deutsche Literatur, n 6, 1992, p. 75-105, ici p. 97).

${ }^{18}$ Cf. Baudrillard, Kool Killer, p. 6.

19 « sie unterwandert mit diesem Trugbild die Wirklichkeit ». C'est nous qui soulignons («Ich» 24).

20 « wir sind im Besitz der alleinigen Wahrheit » (ibid., 76). 
Il faut comprendre ici, non la découverte (par exemple scientifique) de la vérité, mais sa production. En conséquence, ironise Feuerbach, le pouvoir continuera d' " envoyer sous les verrous les gens qui ne veulent pas croire à nos discours grandiloquents ${ }^{21}$ ». Ces propos - sous la plume de l'auteur - disqualifient la parole officielle comme rhétorique de l'imposture.

L'officier de la Sécurité, Feuerbach, fabrique des « faux » à l'insu du narrateur, devenu l'IM-Cambert. C'est ainsi que ce dernier découvre, des années plus tard, que sa mère recevait régulièrement des lettres tapées en son nom, sur sa propre machine à écrire, avec sa signature parfaitement imitée, en provenance de différentes villes ouest-allemandes, qui faisaient croire qu'il vivait en RFA alors qu'il se trouvait à Berlin-Est ( $I c h » 375)$. Il apprend aussi que Cindy s'est rendue à un rendezvous qui lui fut donné en son nom et qu'elle s'est fait arrêter («Ich» 306, 328). La Sécurité échafaude par ailleurs des mises en scène dont il serait l'acteur pour juguler les candidatures au départ dans les délégations étrangères («Ich»178): il devrait, par exemple, feindre de se réfugier dans une délégation étrangère à Berlin, en bleu de travail, y déclarer son désir de quitter la RDA, puis se raviser publiquement par crainte, dirait-il, de perdre les bonnes conditions de vie qui lui sont assurées à l'Est. Ces supercheries diverses culminent avec l'énorme mensonge sur la nature même de sa mission: le poète underground «Reader» qu'il est chargé d'espionner est lui-même un IM. En le surveillant, il le crédite du statut de dissident, avant son transfert à l'Ouest où ce dernier pourrait tranquillement espionner les artistes qui ont fui la RDA. Le narrateur sert en réalité d'appât pour " fabriquer » un faux contestataire. Cette révélation en fin de récit oblige personnage et lecteur à relire à l'envers tout le parcours précédent (fictif et narratif). L'un et l'autre ont été « dupés ».

Hilbig multiplie les références aux thèses de Baudrillard dans son roman pour signifier l'érection du mensonge en praxis dominante: le narrateur affirme à chaque nouvelle étape de sa vie d'informateur avoir vécu ou vivre dans « la simulation ». Par exemple, lorsque le narrateur, devenu l'IM-Cambert, résume sa nouvelle situation:
Ich lebte in einer Welt der Vorstellung [...] immer wieder konnte es geschehen, daß mir die Wirklichkeit phantastisch wurde, irregulär und von einem Augenblick zum anderen bestand die Ruhe für mich nurmehr in einer unwahrscheinlich haltbaren Simulation [...]. $(« I c h » 44)$

Face à l'écart programmé entre les discours officiels et la réalité, les représentants du pouvoir vivent au cœur de la simulation, dans la dualité et l'absurdité:

\footnotetext{
Wir betrieben ununterbrochen Aufklärung, inwiefern sich die Wirklichkeit unseren Vorstellungen schon angenähert hatte [...] wir glaubten unseren Vorstellungen nicht, denn wir klärten ununterbrochen auf - für uns selber! -, daß es keinen Grund gab, ihnen Glauben zu schenken, den Vorstellungen. Aber es war schwer, aufzuklären ohne eine Vorstellung davon, was durch Aufklärung sichergestellt und gegebenenfalls verhindert werden sollte, möglichst im Ansatz schon verhindert werden sollte, wie es unser ausdrückliches Ziel war. Darum war es notwendig zu simulieren, daß die Wirklichkeit unseren Vorstellungen entsprach. («Ich» 44-45)
}

21 « unser bombastisches Geschwätz » (ibid., 77). 
Passons sur les variations sémantiques des termes «Vorstellung(en)» - entre « imaginaire », « idées » ou « vision des choses » -, et «Aufklärung » - qui était un fondement du discours de légitimation en RDA et qui se trouve ironiquement détourné de ses origines historique et philosophique. Mais notons encore qu'il est difficile de déterminer, dans cet univers irréel de duplicité, si l'objet de ces « explications » («Aufklärungen »), comme le note le narrateur, relève « encore » de la simulation ou « déjà » de la réalité :

[O]b sie noch in den Bereich der Simulation gehörten, ob sie schon im Ansatz Wirklichkeit geworden waren. ( $I$ Ich» 45) 22

Sur le même mode ironique, une dizaine d'années plus tôt, Volker Braun pointait une rhétorique du camouflage dans les discours des dirigeants politiques, qui dissout les difficultés ou les échecs du présent dans les promesses d'un avenir proche (sur le modèle encore/ bientôt - hier/ demain) pour fonder le «mensonge » et la ruse comme méthode de gouvernement:

Kunze sagte nicht: Wir sind im Rückstand, er sagte: Wir müssen das Tempo erhöhen. Er sagte nicht: Da wurde ein Fehler gemacht, er sagte: Vorwärts zu neuen Erfolgen. ${ }^{23}$

La perplexité qui envahit le récit ouvre et ferme l'ensemble du texte, puisqu'on retrouve le même motif en position exacte de miroir à la fin du roman:

Ich lebte in einer Welt der Vorstellung [...] in einer unhaltbaren Simulation. ( I Ich» 45)

[I]ch lebte in der Simulation, ich lebte in einem Land voll von simulierter Konsequenz.

$(« I c h » 335)$

Un tel enchâssement - ou plus précisément une telle épanadiplose (c'est-à-dire une répétition en forme de chiasme d'un groupe de mots du début vers la fin) symbolise de façon précise l'autoréférentialité du discours public et, au-delà, le déclin partagé du personnage et du pays/ système.

L'auteur confie ici sa propre analyse sur le système au personnage central:

Die DDR [war] in dem, was sie vorgab, eben nicht existent, sie war eine Simulation, jedenfalls ihr Charakter, den sie proklamierte, eine Simulation. ${ }^{24}$

La police politique poursuit moins une subversion réelle qu'elle ne l'invente, allant jusqu'à l'étendre à toute la population: dans cette logique, le système n'existe que pour déjouer des complots réels ou « imaginaires ». « Nous contribuons à maintenir le système ", s'impatiente Feuerbach devant l'absence de révolte ouverte chez les intellectuels depuis l'affaire Biermann, « et qu'est-ce qu'un système qui n'a pas d'adversaire ${ }^{25} » ?$ « Pensez-vous, ajoute-t-il, que ça nous fasse plaisir d'être sans cesse

\footnotetext{
${ }^{22}$ C'est nous qui soulignons.

23 Volker Braun, Berichte von Hinze und Kunze, Frankfurt a. M., Suhrkamp, 1983, p. 8.

${ }^{24}$ Wolfgang Hilbig « Die Abwesenheit als Ort der Poesie », entretien avec Werner Jung, Neue Deutsche Literatur, ${ }^{\circ} 4,1994$, p. 16.

${ }^{25}$ « wir sind systemerhaltend, und was ist das für ein System, das keinen Widerpart hat» («Ich» 204).
} 
obligés d'édifier un nouvel adversaire ${ }^{26} »$ ? Ou encore: quand les « faits » font défaut, il faut les inventer ( $" I c h » 224)$.

L'exemple le plus simple de cette stratégie politique consiste à repérer des candidats au départ, ou à défaut à en décréter, en posant l'hypothèse que cette question doit nécessairement agiter toute la population: de la "préposée aux toilettes » jusqu'aux députés de la Chambre du peuple. Ne pas en manifester le désir relèverait par conséquent de la "provocation » et constituerait déjà en soi un délit. De deux choses l'une, selon le MfS : ou on n'en parle pas, mais on y pense, ou on affirme vouloir rester, mais c'est nécessairement louche. De sorte que les organismes chargés de faire la lumière sur les pensées secrètes des citoyens de ce pays n'envisagent qu'une seule question: est-il vrai que tous ceux qui se taisent sur ce sujet « planifient leur passage imminent de l'autre côté de la frontière »? Dans ce cas, s'interrogent-ils, comment se fait-il qu'ils se taisent avec autant d'obstination $(« I c h » 155)$ ?

De façon plus générale, il s'agira de définir la notion de « délit » de telle manière que tout le monde, mais absolument tout le monde, puisse ainsi tomber sous le coup de la loi et faire alors l'objet de chantage. Dans cette nouvelle « utopie », chacun est potentiellement coupable et devient objet de défiance et de surveillance. L'univers narratif de Hilbig, en s'appuyant sur une réalité historique, prend une dimension hallucinatoire.

On le voit, il ne s'agit pas du tout de surveiller et d'espionner afin de découvrir une vérité ou des activités secrètes qui seraient dangereuses pour l'ordre public. Il s'agit de construire un réseau d'observations et de délations au service d'un modèle qui précède la quête de renseignements. Nous sommes dans la situation, énoncée par Baudrillard, où la simulation - comme modèle de représentation d'un pouvoir discrétionnaire - précède la collecte des informations. On passe de la dis-simulation (du mouchard) à la simulation comme système. Comme le note Baudrillard dans Simulacres et simulation: "Dissimuler est feindre de ne pas avoir ce qu'on a. Simuler est feindre d'avoir ce qu'on n'a pas. »

Je corrigerais ici en remplaçant avoir par être : dissimuler - pour le mouchard est feindre d'être ce qu'on n'est pas. Et Baudrillard de conclure:

Donc, feindre, ou dissimuler, laissent intact le principe de réalité: la différence est toujours claire, elle n'est que masquée. Tandis que la simulation remet en cause la différence du "vrai" et du "faux", du "réel" et de "l'imaginaire". ${ }^{27}$

Dans le roman, le siège du mensonge d'État est localisé, longuement décrit et commenté par le narrateur lors d'une brève incursion dans le bâtiment du MfS à l'angle de la Normannenstraße et de la Ruchstraße. Le lieu est repérable, il a une réalité historique:

${ }^{26}$ « einen neuen Widerpart aufbauen zu müssen » (ibid., 204). Heiner Müller a donné une version comique de cette perversion: Heiner MüLlER, « Kentauren » (1988), in Die Schlacht, Wolokolamsker Chaussee, Berlin, Verlag der Autoren, 1988, p. 59-65 (« Der Staat ist eine Mühle die muß mahlen/ Der Staat braucht Feinde wie die Mühle Korn braucht/ Der Staat der keinen Feind hat ist kein Staat mehr», p. 60).

${ }^{27}$ BaUdrillard, Simulacres et simulation, p. 12. 
Es waren hier die gewaltigen Areale von Neubauten [...] direkt über mir begannen sie in ihrer unübersichtlichen Vielzahl und Verschachtelung: sie waren unzugänglich für beinahe jedermann.

Il aperçoit un dédale de cours intérieures qui sont entourées de bâtiments à plusieurs étages empilés comme des boîtes. Il se sent observé par mille regards dissimulés:

Es war Abend, und doch fühlte ich mich beobachtet, und ging gemächlich auf und ab in dem Bestreben, mich möglichst harmlos zu verhalten zwischen diesen vieläugigen Wänden [...] hoch über mir verfärbte sich der Himmel zu düsterem Violett, Scharen von Krähen jagten schreiend darin umher [...] da fragt man sich sofort, ob man je wieder rauskommt, da drin, sagte ich. («Ich» 31-32)

Cette description, placée au début du roman, est à lire comme une métaphore spatiale de la société de surveillance où sont plongés tous les personnages. Elle reproduit la machine panoptique de Bentham qui symbolise, selon Michel Foucault, la société disciplinaire moderne:

Cet espace clos, découpé, surveillé en tous points, où les individus sont insérés en une place fixe, où les moindres mouvements sont contrôlés, où tous les événements sont enregistrés, où un travail ininterrompu d'écriture relie le centre et la périphérie, où le pouvoir s'exerce sans partage ${ }^{28}$.

Mais, si le mensonge fait partie des «technologies de pouvoir» (Foucault), si l'espion est à son tour espionné par mille regards dissimulés, le siège de ces dispositifs, ici localisé, reste un point aveugle. La désignation, pourtant précise par l'auteur, des opérations de pouvoir ne conduit pas à l'identification de ce pouvoir, qui reste une simulation. Comme chez Foucault, je reprends ici un commentaire de Jacques Rancière, le pouvoir reste une énigme ${ }^{29}$. Il n'a pas de « réalité » ni de « vérité ».

\section{L'univers diégétique du personnage}

Y aurait-il cependant, derrière les contre-vérités d'État, une vérité du personnage? La réponse, négative, se décline en deux temps : comme écrivain et comme espion.

Dans un récit analeptique, le narrateur W. raconte comment autrefois il observait la nuit, seul, tapi à l'ombre des buissons, les habitants dans leurs appartements éclairés. Gêné par le double vitrage et le voile des rideaux tirés, il ne comprenait pas les phrases souvent inaudibles qui y étaient prononcées. Et dans la lueur des lampes électriques et la phosphorescence des écrans de télévision, les mots prenaient alors « une tout autre signification ». N'ayant aucune idée du sens de ces discours, il cherchait à reconstituer leur signification à partir des gestes et du mouvement des lèvres. En fin de compte, il décidait de « remplacer les phrases inaudibles prononcées à l'intérieur par d'autres qu'il puisait dans sa tête ${ }^{30} »$. Il est à cette époque encore chauffagiste

\footnotetext{
${ }_{28}$ Michel Foucault, Surveiller et punir, Paris, Gallimard, 1975, p. 228-267, ici p. 230.

29 Jacques Rancière, La Méthode de l'égalité, Paris, Bayard, 2012, p. 75-76.

30 « es blieb ihm nichts übrig, als die ungehörten Sätze aus dem Innern der Räume durch solche aus seinem Kopfe zu ersetzen » («Ich»126).
} 
à l'usine, occupé par ses travaux d'écriture. Et, bien que recruté récemment par la Stasi, il ne semble pas encore exercer sa nouvelle activité d'espionnage, même si, à l'usine, ses collègues le suspectent de rédiger des rapports pour la Sécurité, alors qu'il prend simplement des notes pour ses travaux littéraires personnels. L'auteur se garde de clarifier son statut exact entre écrivain encore ouvrier ou ouvrier déjà espion. Cette indétermination, recherchée par l'auteur, nous autorise à privilégier ici la quête d'inspiration de l'écrivain à partir de l'observation de situations concrètes.

L'auteur installe subtilement un décrochage en plusieurs étapes.

On note d'abord que l'écrivain amateur complète les lacunes dans sa perception du réel en faisant appel à son imagination. Mais progressivement, il opte pour le procédé de substitution d'un contenu et d'un sens fictifs indépendamment du contenu et du sens réels des phénomènes observés:

Freilich ging es ihm anfänglich darum, das wirklich Gesagte zu erkennen [...] später machte er die Erfahrung, daß man sich sehr gut darauf einigen konnte, daß es um das wirklich Gesagte überhaupt nicht ging. Das wirklich Gesagte war in der Regel sowieso unter einer oder mehreren Lagen von banalem Gewäsch versteckt. ("Ich»126-127) ${ }^{31}$

Cette fois, on passe de la perception acoustique (das wirklich Gesagte [1], c'est ce qui a été effectivement dit, mais resta inaudible) à un sens dissimulé derrière des propos anodins (das wirklich Gesagte [2] signifie ce que les locuteurs ont voulu dire à mots couverts). Dans un troisième temps, il s'agira de dégager - entendons reconstituer, inventer - ce sens prétendument caché :

Da er jetzt die Sätze nicht mehr um ihrer Mitteilung willen aufnahm, sondern weil er einen in einem dunklen Bereich hinter ihnen verborgenen Sinn suchte [...] war alles Sprechen für ihn nach und nach zu einer Verschwörung geworden. («Ich»130) ${ }^{32}$

On pourrait objecter que notre écrivain en herbe se met à distance du réel pour mieux le décrire et en dégager la signification profonde, que la fiction distord nécessairement le réel pour en dégager des vérités enfouies. Pour citer Hegel, dans son Introduction à l'esthétique: "Loin d'être, par rapport à la réalité courante, de simples apparences et illusions, les manifestations de l'art possèdent une réalité plus haute et une existence plus vraie ${ }^{33}$. »

Or, ici, il en va tout autrement. "L'écrivain » épie des conversations, non pour découvrir une "vérité ", mais pour prêter à leurs auteurs une pratique conspirative imaginaire, des intentions élaborées ailleurs, dans les cuisines du pouvoir. Comme le mouchard, l'écrivain reste étranger au peuple, extérieur au réel. L'écrivain du roman, que Hilbig décrit finalement comme un écrivain raté, médiocre ( $I c h » 232$ ), dépossédé en partie de ses textes, et dont le succès à venir est dû plus à l'intervention secrète de la Stasi qu'à son talent («Ich» 201), mêle de plus en plus, par la suite, ses rapports de police et ses essais littéraires, en mixant les genres ( Ich» 208).

Quant à l'espion, dont la tâche consiste théoriquement à transmettre des renseignements sans les interpréter, il se met un jour, par jalousie, à falsifier ses

\footnotetext{
31 C'est nous qui soulignons.

${ }^{32}$ C'est nous qui soulignons.

33 Georg Wilhelm Friedrich Hegel, Introduction à l'esthétique, trad. par Samuel JankéLÉvitch, Paris, Aubier, 1964, p. 38-39.
} 
rapports sur l'écrivain Reader, à inventer des rencontres et des conversations qui n'ont pas eu lieu pour noircir son rival et faciliter son propre passage à l'Ouest.

L'un et l'autre, l'écrivain et l'espion, s'installent finalement dans l'imposture et le mensonge.

À un journaliste qui lui reprochait de mettre dans ce roman fiction et espionnage sur le même plan, Hilbig répondit:

\begin{abstract}
Nicht gleichgesetzt, sondern verglichen. Das war eine beklemmende Idee. Ich hatte den Eindruck, daß sich beide Tätigkeiten ähneln. Beide, der Spitzel und der Schriftsteller, schaffen sich eine Wirklichkeitsfiktion und eine Fiktion von Figuren, die sie überdenken, ausloten, observieren. ${ }^{34}$
\end{abstract}

Dans son roman, Hilbig confère aux thèses de Baudrillard sur la simulation un double statut. S'il le convoque pour mettre en scène l'écart qui oppose un discours des dominants fondé sur l'imposture et la "dépossession de la parole » chez les dominés (Bourdieu), il remet en cause la « résignation» (W. Hilbig) des écrivains du Prenzlauer Berg, qu'il fréquentait avec une certaine réserve. Or ces derniers se prévalaient (aussi) des thèses de Baudrillard sur la simulation et des analyses sur l'autoréférentialité du langage chez les poststructuralistes français, pour décréter la disparition du sujet dans la littérature d'avant-garde et justifier leur refus de toute confrontation avec la parole officielle en s'adonnant à des jeux de déconstruction même si on peut y voir une attitude " politique ${ }^{35}$. Dans son roman «Ich », Hilbig choisit de réintroduire le sujet, mais il s'agit d'un personnage fragile à ses yeux, dont la fragilité et les frustrations favorisent la manipulation, un sujet condamné à «l'inauthenticité ${ }^{36}$ », autant victime que coupable. À travers ce personnage, Hilbig expérimente aussi, hors de toute moralisation, les contradictions, voire les compromissions et les impasses d'une littérature de la résignation ${ }^{37}$.

34 Wolfgang Hilbig, « Die DDR-Literatur hatte völlig resigniert », entretien avec Karim SAAB, in Wiтtsтоск, Materialien zu Leben und Werk, p. 222-228, ici p. 224.

35 Voir à ce sujet le témoignage et l'analyse de Jan FAKTOR, « Sechzehn Punkte zur Prenzlauer-BergSzene », in Böthig et MichaEL, MachtSpiele, p. 91-111.

36 « ein kommissarisches Ich » ( Ich» 5).

37 Voir les propos sévères de Hilbig sur cette question: HiLbig, Abriss, p. 53-55. 